Questions de communication

$24 \mid 2013$

Renouvellement des mises en scène télévisuelles de la politique

\title{
Sarah SEPULCHRE, dir., Décoder les séries télévisées
}

De Boeck, coll. Info Com, 2011, 256 pages

Frédéric Pugnière-Saavedra

\section{OpenEdition}

\section{Journals}

Édition électronique

URL : http://journals.openedition.org/questionsdecommunication/8823

DOI : 10.4000/questionsdecommunication.8823

ISSN : 2259-8901

\section{Éditeur}

Presses universitaires de Lorraine

Édition imprimée

Date de publication : 31 décembre 2013

Pagination : 304-306

ISBN : 978-2-8143-0182-5

ISSN : 1633-5961

\section{Référence électronique}

Frédéric Pugnière-Saavedra, «Sarah sepulchre, dir., Décoder les séries télévisées 》, Questions de communication [En ligne], 24 | 2013, mis en ligne le 01 février 2014, consulté le 22 septembre 2020. URL : http://journals.openedition.org/questionsdecommunication/8823 ; DOI : https://doi.org/ 10.4000/questionsdecommunication.8823 
d'un texte) lors de l'examen d'un corpus de journaux d'entreprise. Dernière contribution de ce chapitre «Du récit de contrôle social à la narration éthique: comment se raconte la secte? 》 (pp. 173-186) d'Arthur Mary répond à la question « comment raconte-t-on la secte? »». Ceci grâce à l'étude de deux textes écrits par un ancien témoin de Jéhovah et de la narration par l'auteur même d'un reportage sur les sectes, de son expérience de montage rhétorique, sans qu'il reconnaisse pour autant son propre recours au storytelling.

La dernière contribution est celle de Daniel Moati (pp. 187-200). Elle est présentée sous la forme d'un témoignage dans lequel il évoque « La communication narrative de l'histoire de l'écriture comme vecteur de remédiation esthétique $»$. Outre raconter son expérience personnelle, l'auteur vise à lancer l'espoir que le «storytelling pédagogique, loin de servir à formater les enseignants, puisse aider ces derniers à renouer avec les élèves des classes populaires » (p. 197).

Enfin, l'ouvrage est agrémenté d'une courte biographie de chacun des auteurs et les contributions disposent toutes de riches bibliographies qui entrâneront sans nul doute les lecteurs intéressés sur de nouvelles pistes de réflexion. Dans l'ensemble, Le storytelling. Succès des histoires, histoire d'un succès n'est pas un ouvrage à la portée des lecteurs à la recherche d'une introduction au domaine. II est préférable d'avoir des notions de narratologie et d'avoir lu le livre de Christian Salmon qui y est mentionné. En revanche, les spécialistes y trouveront leur compte.

Justine Houyaux Université de Mons, B-7000 justine.houyaux@umons.ac.be

\section{Sarah SEPULCHRE, dir., Décoder les séries télévisées. De Boeck, coll. Info Com, 20I I, 256 p.}

Préfacé par Éric Maigret, l'ourrage est un recueil de sept contributions réparties en autant de chapitres. Très didactique, il s'adresse tant aux étudiants de licence/ master qu'aux enseignants praticiens du secondaire, ce qui justifie la présence d'outills paratextuels utiles tels une bibliographie complémentaire à la fin de chaque chapitre, des encadrés portant sur les notions importantes, une exemplification à partir de séries actuelles ou plus anciennes, un glossaire, une webographie, une riche bibliographie plus générale et un index par auteur et par série. Bien que les intentions affichées de Sarah Sepulchre soient humbles telles « proposer plusieurs angles d'attaque de la série » (p. 7) et aller contre lidée selon laquelle les séries « lobotomisent le téléspectateur » et sont de la « sous-culture 》 (p.9), elles sont parfaitement lisibles dans les résultats qui, d'ailleurs, vont au-delà car l'ouvrage fournit non seulement des clés d'interprétation, mais suscite l'envie de regarder les séries non plus en tant que spectateur, mais en tant qu'analyste.

Marjolaine Boutet (pp. I I-43) commence l'ouvrage par un chapitre intitulé « Histoire des séries télévisées 》 et dresse un panorama historique en distinguant quatre périodes (ou décennies) : «l'âge d'or 》 des séries américaines (années 50) correspondant au moment où la télévision devient un média de masse et où les formats (western, sitcom, fantastique...) se mettent en place ; « l'âge classique » (années 60-70) voyant une stabilisation et un perfectionnement de ces formats - pour plaire à l'Américain moyen à travers une orientation forte vers le divertissement (naissances des séries judiciaires, médicales) - lesquels traitent de thèmes de société tels la Guerre froide, les conquêtes spatiales, les inégalités entre Noirs et Blancs, les femmes, la critique de la guerre du Vietnam; ;e « deuxième âge d'or des fictions télévisées » (années 80) coïncidant avec les arrivées de la technologie (télécommande, magnétoscope, chaines câblées) et des baby-boomers à l'âge adulte ainsi qu'avec une grande capacité inventive dans les formes de série (dites « addictives》, « chorales 》, « réflexives 》) ; « l'ère numérique » (années 90-2000), période pendant laquelle l'offre se multiplie, les audiences s'éparpillent et l'acte de regarder la télévision devient une expérience individuelle et non plus une activité se limitant à séduire le grand public.

Séverine Barthes (pp. 47-73) poursuit l'ouvrage avec un chapitre intitulé « Production et programmation des séries télévisées » présentant le secteur télévisuel privé étasunien composé de réseaux, de chaînes locales, de chaînes du câble et du satellite et de chaînes premium où ce sont les logiques d'affrontements économiques qui déterminent le cycle de vie des séries qu'il s'agisse de séries dites « daytime soap opera 》 ou de séries dites de « fiction de soirées 》 (p. 62). Au cœur des enjeux de programmation des séries, ce constat implique des stratégies différentes de programmation : logique d'accompagnement des activités quotidiennes pour les premières et logique d'adhésion aux contenus proposés pour les secondes. Elles impliquent également de suivre aussi bien le rythme de la vie quotidienne que des usages sociaux et culturels hebdomadaires du public. Ces logiques de programmation ont pour conséquence de créer chez le téléspectateur de la fidélisation, des habitudes avec l'effet d'agenda où se superposent temps de fiction et 
calendrier de téléspectateur, avec différents effets de générique, des spin off, des crossover dans un contexte difficile de productions en flux tendu.

Ensuite, Stéphane Benassi (pp. 75-10I) consacre un chapitre intitulé "Sérialité 》 aux questionnements qu'implique la notion de sérialité en partant du constat selon lequel les formats actuels de fiction télévisuelle n'obéissent plus au genres traditionnels qui remontent aux origines de la télévision (téléfilm, feuilleton et série), d'où la première approche proposée par l'auteur qui distingue la mise en série - « déclinaison d'une matrice de départ qui donne pour fixes un schéma narratif et des paramètres sémantiques et temporels » (p. 78) et la mise en feuilleton - « étirement du récit fictionnel susceptible de subir des variations sémantiques [...] temporelles [...] et narratives » (ibid). Pour éviter les ambiguités de terminologie, l'auteur définit la sérialité comme « désign[ant] l'ensemble des phénomènes susceptibles de générer la fiction plurielle » (p. 79), et poursuit sa réflexion en proposant la notion de sérialité matricielle agissant au niveau de l'œuvre pour définir, qualifier et fixer les invariants du récit, pouvant aboutir à l'émergence de genres principaux tels le feuilleton canonique et le feuilleton sérialisant, d'une part, et la série canonique et la série feuilletonante, d'autre part, à partir respectivement des formes théoriques du feuilleton et de la série. Puis, dépassant le niveau de l'œurre pour se centrer sur le niveau du média, Stéphane Benassi propose la notion de sérialité programmationnelle pour insister sur les processus de contagion sérielle (liés aux phénomènes de mise en module ou en paradigme) ou de contagion feuilletonesque. L'auteur termine le chapitre en insistant sur le caractère prépondérant de la sérialité en tant que liant esthétique entre téléspectateur et fiction.

Sarah Sepulchre (pp. 107-I48) consacre un chapitre au «Personnage en série » et annonce d'emblée l'impossibilité d'offrir en quelques pages « toutes les bases théoriques, toutes les clés de compréhension et tous les outils d'analyse » (p. 107) tant l'objet est complexe. Après un rappel portant sur des considérations générales (le personnage est développé par l'auteur, construit par l'acte de lire ; c'est également une composante essentielle du récit), l'auteure passe en revue les chercheurs littéraires qui ont consacré leurs travaux au personnage du récit (Oswald Ducrot, Tzvetan Todorov, Dictionnaire encyclopédique des sciences du langage, Paris, Éd. Le Seuil, 1972 ; Oswald Ducrot, Jean-Marie Schaeffer, Nouveau Dictionnaire encyclopédique des sciences du langage, Paris, Éd. Le Seuil, 1995 ; Pierre Glaudes, Yves Reuter, Personnages et didactique du récit, Metz,
Metz, université Paul Verlaine-Metz, 1996 ; Horton, Writing the Character-Centered Screenplay, Berkeley/ Londres/Los Angeles, University of California Press, 1999, p. 108) et, parmi eux, elle s'appuiera sur le modèle de Pierre Glaudes et Yves Reuter (op. cit, pp. 108-109) pour appréhender le personnage des séries sous trois angles : le genre, l'organisation textuelle et l'investissement affectif. Le personnage permet donc de « faire avancer le récit, $[. .$.$] est le vecteur$ des valeurs de l'auteur et du lecteur » (p. 117). S'inspirant ensuite des pistes concernant le système de personnages développées par Philippe Hamon (Le Personnel du roman. Le système des personnages dans Les Rougon-Macquart d'Émile Zola, Genève, Droz, 1983, p. I 10) la responsable de l'ouvrage avance que c'est par l'opposition de six procédés différentiels (pp. I|8-1|9) que le téléspectateur distinguera le personnage principal des personnages secondaires et qu'il dégagera des éléments de qualification (le portrait d'un personnages place le téléspectateur dans un horizon d'attente) et de fonctionnalité (les actions du personnage). Les séries télévisées ont complexifié le système des personnages avec un plus grand nombre de héros, des personnages secondaires multiples et des personnages stéréotypés.

Par la suite, dans le chapitre intitulé « Comment analyser les publics des séries télévisées 》, Laurence Doury (pp. 151-174) décrit à grands traits l'évolution des recherches sur le public des médias tout au long du $x x^{e}$ siècle. Après une première période pendant laquelle les médias manipulent le public de masse (notions de foules et de masse), apparait une seconde où les publics sont capables de donner du sens aux médias et de les interpréter dans le sillon des Cultural studies. L'auteure poursuit avec les différents outils (techniques d'enquête) dont le chercheur a besoin pour étudier les phénomènes de réception : observation de téléspectateurs, entretiens avec les téléspectateurs (individuels/collectifs) et questionnaires. Elle achève son chapitre par une application concrète à partir de l'étude de séries policières américaines débouchant sur la mise au jour d'un certain nombre d'invariants : visée divertissante, fonction pédagogique, histoire captivante permettant de sortir du quotidien.

Dans le chapitre intitulé « Récits cumulatifs et arcs narratifs》, Ursulra Ganz-Blaettler (pp. 179-191) débute par quelques considérations sur la narration sérielle (et donc sur la question du temps) de l'ère de la transition à l'ère post-réseaux. L'auteure poursuit par l'examen de deux procédés apparus à l'ère où les techniques de programmation, les stratégies des chaînes et les habitudes de visionnages nouvelles rendent possible le 
fait de revenir sur les saisons antérieures : procédé dit de l'arc narratif qui se développe sur plusieurs épisodes et le procédé dit des blessures du passé (vécues par un/ des personnages) qui modifient à la fois le déroulement du temps (prolongement possibles vers le passé ou vers le futur) et la mémoire diégétiques dans les soap operas. Ces deux procédés facilitent le lien entre le contexte, les récits et les usages spectatoriaux.

Dans le dernier chapitre, intitulé « Séries télévisées et réalités : les imaginaires sériels à la poursuite du réel » (pp. 193-21 I), et après un prologue visant à lever les ambiguités sémantiques sur les mots clés du titre série télévisée et réalité, Jean-Pierre Esquenazi fournit des indications sur le réseau sémantique distinguant le réel, du symbolique et de l'imaginaire. II précise ensuite la nature des relations entre le récit fictionnel et le réel la fiction peut être une « paraphrase du réel » (p. 198), peut se rapporter à la représentation de la réalité inspirée par un monde historique précis, contemporain ou non (p. 199) et peut être appropriée par le récepteur et par le monde du destinataire (p. 200), ce qui revient à considérer à travers de nombreux exemples les différents niveaux qu'entretient la série avec le réel : ceux des créateurs, du contexte social et des attentes spécifiques et celui du récepteur.

En conclusion, ces sept contributions - de difficultés de lecture différente - convergent parfaitement pour donner des clés d'analyse des séries télévisées. Toutefois, on peut regretter que le chapitre de Laurence Doury n'évoque pas la tendance nouvelle consistant à étudier le comportement des téléspectateurs dans un environnement dédié à l'observation expérimentale (oculomètre ou eyetracker), ce qui renouvelle la nature des contraintes qu'impose l'outil et revisite la méthodologie de la recherche sur la réception des programmes à la télévision. Pour autant, ce bémol ne diminue en rien le réel intérêt de l'ouvrage.

Frédéric Pugnière-Saavedra

Prefics, université de Bretagne-Sud, F-56000 frederic.pugniere-saavedra@univ-ubs.fr

\section{Jean STERN, Les patrons de la presse nationale. Tous mauvais.} Paris, Éd. La Fabrique, 2012,210 p.

Solidement documenté, l'ouvrage se situe dans la lignée de publications engagées qui, depuis des lustres, réunissent informations pertinentes et tonalité peu amène avec l'objet scruté - ici, les patrons de presse. L'épilogue capture ce ton avec l'emploi d'allusions - «Tous à la ferme » (p. 169) - au discours managérialpublicitaire qui fait des ravages dans les médias depuis un demi-siècle. « La ferme des contenus » (ibid.), quant à elle, est liée au développement des sites internet depuis les années 90 et les agrégateurs numériques des moteurs de recherche. Les « attentes de la multitude » (ibid.) façonneraient la production des breaking news par tout un chacun, les journalistes dits professionnels se trouvant ainsi broyés par l'abondance des producteurs de «l'info » et de ses produits dérivés.

En effet, le sort de journalistes se situe en filigrane de l'enquête conduite par le journaliste. Les patrons des groupes de presse/médias - « Pigasse-LagardèreRothschild-Dassault-Niel-Arnault » (p. 109) - tirent leur épingle du jeu, s'enrichissent souvent, non sans risque ; les journalistes, eux, restant souvent sur la paille, de plus en plus fragilisés. La rengaine est connue mais n'en est pas moins vraie, même s'il est des journalistes devenus eux-mêmes cadres dirigeants. Ceci est largement attesté dans un ouvrage nourri tant par des informations récentes, recueillies auprès de nombreux sources et sites, que par des « infos » puisées dans des volumes, souvent rédigés par des journalistes des années 70-80, dénonçant les « trois $H$ [...] qui « mont[ai]ent en puissance » (p. 47) alors - les Havas, Hachette, Hersant, même si les deux premiers avaient déjà entamé leur ascension dès le milieu du XIXe siècle.

Jean Stern démonte les avantages des divers holdings, des stratégies déployées par des groupes aux intérêts nombreux, des liaisons dangereuses entre ce patronat de presse, des médias, souvent présent dans d'autres branches des industries culturelles - qu'on voudrait rebaptiser « industries créatives 》... - et des hommes politiques au pouvoir, sans parler des banques. Après les liens connus entre François Mitterrand et Robert Hersant, on revisite ceux entretenues entre Nicolas Sarkozy et François Pinault. Publié en 2012, Les patrons de la presse nationale ne traite pas de l'arrivée à la tête du quotidien Le Monde, en 2013, de Natalie Nougayrède - journaliste-maison - et de Vincent Giret - précédemment directeur délégué de la rédaction de Libération. Mais, plusieurs des thèmes - par exemple, l'importance du site internet du journal et le déclin de la version papier - se situeraient en arrière-fond.

Dans un court passage, Jean Stern, sorti du Centre de formation des journalistes (CF) en 1977, évoque son propre parcours de journaliste et indique que le trajet à l'origine du « naufrage... de la presse nationale » motive son essai.

En conclusion, qu'il nous soit permis de poser une question dérangeante : que font de tels ouvrages les enseignants-chercheurs en communication dispensant, 